\title{
Zum Jahresbericht 2008 des Schweizerischen Toxikologischen Informationszentrums Vergiftungen in der Schweiz
}

Hugo Kupferschmidt

Korrespondenz:

Dr. med. H. Kupferschmidt Direktor Schweizerisches Toxikologisches Informationszentrum (STIZ) Freiestrasse 16 CH-8032 Zürich

hkupferschmidt@toxi.ch
Das Tox-Zentrum führte 200833366 Beratungen durch. Dabei handelte es sich in über $83 \%$ um Anfragen zu Giftexpositionen, die übrigen Anfragen waren prophylaktischer oder theoretischer Natur. Knapp drei Viertel aller Anfragen bezogen sich auf Medikamente, Haushaltprodukte oder Pflanzen (Tab. 1).

54,1\% der Expositionen fanden bei Kindern statt, mehrheitlich im Vorschulalter (80,1\% aller Expositionen bei unter 16-Jährigen bei Kindern $<5$ Jahren). Bei der Geschlechtsverteilung war bei den Kindern ein leichtes Überwiegen der Knaben (49,6\% vs. 43,8\% Mädchen) und bei den Erwachsenen der Frauen (57,5\% vs. $40 \%$ Männer) zu beobachten. $91 \%$ der etwas über 21000 unbeabsichtigten (akzidentellen) Vergiftungen ereigneten sich im häuslichen Milieu, bei den ca. 5300 beabsichtigten Intoxikationen trat die grösste Anzahl (72,6\%) im Rahmen von Suizidversuchen auf.

\section{Schwere und tödliche Vergiftungen in der Schweiz 2008}

Von 256 schweren Vergiftungen mit Medikamenten (davon 16 Kinder) betrafen 213 Mittel für das Nervensystem, im Wesentlichen Analgetika (Opioide, Paracetamol), Antiepileptika (Phenobarbital, Carbamazepin) und Psychopharmaka (Benzodiazepine $\mathrm{n}=55$, Antidepressiva $n=57$, Antipsychotika $n=37$, Zolpidem, Zopiclon, Zaleplon $\mathrm{n}=16$ ). Bei den neun Todesfällen durch Medikamente waren in suizidaler Absicht Doxepin, Fentanyl, Trimipramin, Venlafaxin, Verapamil, Cilazapril, Hydrochlorothiazid, Ibuprofen, Zolpi-

\section{Empoisonnements en Suisse en 2008}

En 2008, le Centre suisse d'information toxicologique (CSIT), communément appelé le «Tox» a mené 33366 consultations. $83 \%$ des demandes concernaient l'exposition à des produits toxiques. Le reste des conseils prodigués était de nature prophylactique et théorique. Trois quarts des demandes étaient liés à des médicaments, des produits ménagers ou des plantes.

$54,1 \%$ des expositions se sont produites chez les enfants, le plus souvent avant l'âge scolaire $(80,1 \%$ des expositions chez les moins de 16 ans concernaient des enfants de moins de 5 ans). Chez les enfants, on constate une légère prédominance des garçons ( $49,6 \%$ contre $43,8 \%$ chez les filles) et à l'âge adulte, une prédominance des femmes $(57,5 \%$ contre $40 \%$ chez les hommes). $91 \%$ des 21000 empoisonnements non intentionnels (accidentels) se sont produits en milieu domestique. 5300 représentent des intoxications volontaires, dont la majeure partie $(72,6 \%)$ s'est produite dans le cadre de tentatives de suicide.

\section{Tabelle 1}

Häufigkeit der Vergiftungen beim Menschen nach Noxengruppen (Beratungen STIZ 2008).

\begin{tabular}{lcccc} 
Noxengruppen/Altersgruppen & Erwachsene & Kinder & Alter undefiniert & Total \\
\hline Medikamente & 5596 & 4479 & 4 & $10079(36,3 \%)$ \\
\hline Haushaltprodukte & 2306 & 4817 & 24 & $7147(25,7 \%)$ \\
\hline Pflanzen & 518 & 2294 & 11 & $2823(10,2 \%)$ \\
\hline Technische und gewerbliche Produkte & 1410 & 387 & 8 & $1805(6,5 \%)$ \\
\hline Körperpflegemittel und Kosmetika & 190 & 1229 & & $1419(5,1 \%)$ \\
\hline Nahrungsmittel und Getränke & 608 & 433 & 17 & $1058(3,8 \%)$ \\
\hline Genussmittel, Drogen und Alkohol & 535 & 441 & & $976(3,5 \%)$ \\
\hline Produkte für Landwirtschaft und Gartenbau & 358 & 370 & 2 & $730(2,6 \%)$ \\
\hline Pilze & 297 & 183 & 7 & $487(1,8 \%)$ \\
\hline (Gift-)Tiere & 242 & 117 & 2 & $361(1,3 \%)$ \\
\hline Tierarzneimittel & 61 & 45 & & $106(0,4 \%)$ \\
\hline Andere oder unbekannte Noxen & 553 & 243 & 15 & $811(2,9 \%)$ \\
\hline Total & 12674 & $\mathbf{1 5 0 3 8}$ & 90 & $\mathbf{2 7 8 0 2 ( 1 0 0 \% )}$ \\
\end{tabular}


dem, Proranolol und Alprazolam, zum Teil in Kombination, eingenommen worden.

Von den 44 schweren Vergiftungen mit Genussmitteln, Drogen und Alkohol betrafen 19 Alkoholvergiftungen, eine Nikotinvergiftung, zwei Halluzinogene und Stimulantien (inkl. Ecstasy), vier Opiate, vier Kokain und 14 Gammahydroxybutyrat (GHB) oder Gammabutyrolacton (GBL). Bei der Nikotinvergiftung handelte es sich um eine betagte demente Frau, die 2-3 gerauchte Zigarrenstummel eingenommen hatte, worauf es zu Erbrechen und einer schweren Hypertonie kam. Ein 14-jähriges Mädchen war komatös nach dem Konsum einer grösseren Menge alkoholischer Getränke. Ein 15-jähriger Knabe wurde nach dem Konsum von Gammabutyrolacton (GBL; Nachweis im Plasma: GHB 224 mg/L) und etwas Alkohol tief komatös, hypotherm und mit einer schweren Bradykardie von 27/min. im Winter mit nassen Kleidern im Freien aufgefunden. Ein 63-jähriger Mann erlitt nach Einnahme von Ecstasy eine Hyperthermie mit Rhabdomyolyse und verstarb an Multiorganversagen.

Expositionen mit Pflanzen führten $2008 \mathrm{zu}$ vier schweren Vergiftungen, eine davon bei einem Kind. Ein 18-jähriger und ein 21-jähriger Mann konsumierten in missbräuchlicher Absicht selbstgebrauten Tee aus Engelstrompete (Datura suaveolens) und erlitten ein schweres anticholinerges Syndrom mit Verwirrung, Sopor, optischen Halluzinationen, intermittierender Agitation, Tachykardie und Mydriasis. Ein 42-jähriger Mann lag nach der Einnahme von 60 Ricinussamen (Ricinus communis), 2,5 g Diphenhydramin, Ibuprofen und Alkohol längere Zeit komatös am Boden; er entwickelte eine Aspirationspneumonie durch Erbrechen, eine Rhabdomyolyse und eine Gastroenteritis mit Darmblutung. Ein 2-monatiges Kind erlitt Krampfanfälle nach Gabe von Tee aus japanischem Sternanis (Illicium anisatum), der mit echtem Sternanis (Illicium verum) verwechselt wurde.

Bei den Haushaltprodukten traten neun schwere Intoxikationen auf, eine davon bei einem Kind. Ein 4-jähriger Knabe erlitt nach der Einnahme eines Teppichreinigungsmittels ösophageale Verätzungen. Bei vier erwachsenen Patienten kam es zu schweren gastrointestinalen Läsionen nach Einnahme korrosiver Reinigungsprodukte oder von Entkalkern in suizidaler Absicht. Ein 37-jähriger Mann kam am Arbeitsplatz längere Zeit mit nasser Asche in Kontakt und erlitt dadurch ausgedehnte Hautverätzungen, die chirurgisch versorgt werden mussten. Ein 34-jähriger Mann entwickelte eine schwere Bronchokonstriktion, nachdem er längere Zeit in einem geschlossenen, ungelüfteten Raum mit Javelwasser (Natriumhypochlorit) gearbeitet hatte. Ein 44-jähriger Patient wurde bei der Arbeit in einem Tank durch Sauerstoffmangel bewusstlos und konnte erst nach längerer Zeit geborgen werden. Er erholte sich vollständig. Ein 30-jähriger Mann verletzte sich bei der Arbeit mit einem Hochdruckreiniger an einem Finger, was eine Nekrose verursachte, die chirurgisch debridiert werden musste.
Kosmetika und Körperpflegemittel führten 2008 nicht zu schweren Vergiftungen. Bei je einem Erwachsenen führte die Einnahme von Shampoo bzw. Parfüm zu mittelschweren Symptomen, und bei einem 13-jährigen Mädchen trat nach der Anwendung von Haartönungsmittel ein schweres, nässendes Ekzem der Kopfhaut auf.

Mit Nahrungsmitteln und Getränken kam es 2008 zu einer schweren Vergiftung: Bei einem 58-jährigen Mann traten Übelkeit, Erbrechen, Bradykardie und Blutdruckabfall auf, nachdem er mit Grayanotoxinen kontaminierten Honig aus der Türkei konsumiert hatte. Bei seiner Ehefrau kam es zu ähnlichen, aber weniger ausgeprägten Symptomen. In einem mittelschweren Fall kam es zu einer Gastroenteritis nach Genuss von Meeresfrüchten.

$\mathrm{Zu}$ sieben schweren und einem tödlichen Vergiftungsfall kam es durch technisch-gewerbliche Produkte: Ein 59-jähriger Mann unternahm einen Suizidversuch, indem er in seinem Wohnwagen Propangas ausströmen liess. Er erlitt eine kurzzeitige Bewusstlosigkeit mit Atemstillstand, konnte aber gerettet werden. Zwei erwachsene Personen tranken Ammoniak, was zu schweren Verätzungen des oberen Gastrointestinaltraktes führte. In drei weiteren Fällen kam es nach Einnahme von starken Laugen zu ausgeprägten Läsionen des Mundes, Rachens, Ösophagus und Magens. Bei den Noxen handelte es sich einmal um einen Kaffeelöffel 30\% Natronlauge, einmal um ein natronlaugenhaltiges Reinigungsmittel (bei einem 3-jährigen Kind), und einmal um einen Melkmaschinenreiniger. Tödlich verlief die Einnahme von $500 \mathrm{ml}$ Methanol unbekannter Konzentration in suizidaler Absicht bei einem 25-jährigen Mann, was zu Koma, einem Krampfanfall und einer schweren metabolischen Azidose (arterieller pH 6,3) führte. Er konnte trotz antidotaler Behandlung mit Ethanol und Fomepizol sowie Hämodialyse nicht gerettet werden.

Stoffe in Landwirtschaft und Gartenbau: Ein knapp zweieinhalbjähriges Mädchen trank eine unbekannte Menge eines Diazinon-haltigen Insektizides (Rosenpflegemittel) und entwickelte ein schweres cholinerges Syndrom mit Nausea, Erbrechen, Miosis, Schwitzen, Hypersalivation, Bronchorrhoe, Diarrhoe, Faszikulationen und einem Krampfanfall. Es wurde mit Atropin und Obidoxim behandelt und erholte sich. Ein 67-jähriger Mann trank in suizidaler Absicht ein ebenfalls Diazinon-haltiges Insektizid. Auch bei ihm zeigte sich eine ausgeprägte, typische cholinerge Symptomatik. Er wurde mit Atropin behandelt und erholte sich vollständig. Zwei Männer mittleren Alters erlitten nach dem Sturz in eine Jauchegrube einen Herzstillstand und mussten reanimiert werden. Bei einem blieben neurologische Spätsymptome im Sinn einer hypoxischen Enzephalopathie zurück, einer war nach sechs Tagen immer noch verlangsamt. Beide wiesen auch Kornealäsionen durch die Jauche auf. Ein weiterer Patient wollte einen der beiden Vorgenannten retten und wurde durch die Wirkung der Jauch- 


\begin{tabular}{|c|c|c|c|c|c|c|c|c|c|c|c|}
\hline \multirow[b]{2}{*}{ Noxengruppen/Schweregrad } & \multicolumn{5}{|c|}{ Erwachsene } & \multicolumn{5}{|c|}{ Kinder } & \multirow[t]{2}{*}{ Total } \\
\hline & 0 & $\mathbf{L}$ & M & $s$ & $\mathbf{T}$ & 0 & $\mathbf{L}$ & M & $S$ & $\mathbf{T}$ & \\
\hline $\begin{array}{l}\text { Medikamente } \\
\text { davon }\end{array}$ & 300 & 1300 & 342 & 231 & 8 & 342 & 245 & 49 & 16 & - & $2833(64,5 \%)$ \\
\hline - Nervensystem & 182 & 1045 & 262 & 197 & 5 & 110 & 132 & 27 & 11 & & 1971 \\
\hline - Atemwege & 12 & 53 & 32 & 1 & & 58 & 45 & 11 & 1 & & 213 \\
\hline - Bewegungsapparat & 38 & 93 & 20 & 8 & & 27 & 20 & 6 & 2 & & 214 \\
\hline - Kreislauf & 17 & 43 & 10 & 12 & 3 & 37 & 13 & 1 & 1 & & 137 \\
\hline - Verdauung & 13 & 21 & 6 & 5 & & 44 & 12 & 1 & 1 & & 103 \\
\hline - übrige & 38 & 45 & 12 & 8 & & 66 & 23 & 3 & & & 195 \\
\hline Haushaltprodukte & 34 & 165 & 30 & 8 & & 109 & 105 & 3 & 1 & & $455(10,4 \%)$ \\
\hline $\begin{array}{l}\text { Technische und gewerbliche } \\
\text { Produkte }\end{array}$ & 35 & 227 & 25 & 6 & 1 & 7 & 26 & 6 & 1 & & $334(7,6 \%)$ \\
\hline $\begin{array}{l}\text { Genussmittel, Drogen } \\
\text { und Alkohol }\end{array}$ & 15 & 124 & 86 & 42 & 1 & 10 & 15 & 6 & 2 & & $301(6,9 \%)$ \\
\hline Pilze & 5 & 27 & 40 & 3 & & 14 & 6 & 1 & 1 & & $97(2,2 \%)$ \\
\hline Pflanzen & 5 & 21 & 10 & 3 & & 27 & 13 & 2 & 1 & & $82(1,9 \%)$ \\
\hline $\begin{array}{l}\text { Produkte für Landwirtschaft } \\
\text { und Gartenbau }\end{array}$ & 6 & 25 & 8 & 6 & 1 & 13 & 2 & & 1 & & $62(1,4 \%)$ \\
\hline Körperpflegemittel und Kosmetika & 13 & 2 & & & 20 & 19 & 1 & & & & $55(1,3 \%)$ \\
\hline (Gift-)Tiere & 1 & 16 & 5 & 4 & & 3 & 10 & 2 & 2 & & $43(1,0 \%)$ \\
\hline Nahrungsmittel und Getränke & 2 & 14 & 1 & 1 & & 3 & 5 & & & & $26(0,6 \%)$ \\
\hline Tierarzneimittel & 4 & 7 & & 1 & & 2 & 1 & & & & $15(0,3 \%)$ \\
\hline Andere oder unbekannte Noxen & 8 & 47 & 10 & 4 & & 6 & 12 & 3 & & & $90(2,0 \%)$ \\
\hline Total & 415 & 1986 & 559 & 309 & 11 & 556 & 459 & 73 & 25 & 0 & $4393(100 \%)$ \\
\hline
\end{tabular}

gase (Schwefelwasserstoff $\mathrm{H}_{2} \mathrm{~S}$ ) ebenfalls bewusstlos und stürzte in die Jauche. Er erholte sich ohne Folgen. Zwei weitere Patienten wurden beim Besteigen eines Silos bewusstlos und stürzten hinein. Einer der beiden musste vor Ort reanimiert werden. Ein Retter verlor ebenfalls kurzzeitig das Bewusstsein. Weniger Glück hatte ein 72-jähriger Landwirt, der ebenfalls komatös im Silo gefunden wurde und einen anoxischen Hirnschaden erlitt, an dem er später verstarb. Bei den toxischen Gasen in Gärsilos, wie sie auf Bauernhöfen vorkommen, handelt es sich um Kohlendioxid und nitrose Gase.

Giftige Tiere: Von 35 Bissen einheimischer Schlangen verliefen zwei schwer. Ein etwas mehr als einjähriges Mädchen wurde von einer Kreuzotter in den Fuss gebissen. Wegen der Schwellung und einer zunehmenden Gerinnungsstörung wurde das Antivenin verabreicht, worauf die Befunde besserten. Ein 12-jähriger Knabe wurde von einer Viper in den Daumen gebissen und entwickelte ausgeprägte Allgemeinsymptome mit Verwirrung, Blutdruckabfall, Schwitzen, Durchfall, Erbrechen und leichter Rhabdomyolyse. Trotzdem erholte er sich auch ohne Gabe des Antivenins. Das STIZ registrierte 2008 zudem 23 Fälle von Bissen exotischer Schlangen. Bei den insgesamt 58 Schlangenbissen waren 18 Kinder und 40 Erwachsene betroffen. In 39 Fällen handelte es sich um Giftschlangen, zehn davon in Haltung (Agkistrodon contortrix, Crotalus atrox, Crotalus durissus unicolor, Crota- lus lepidus, Crotalus sp., Dendroaspis viridis, Hemachatus hemachatus, Naja nivea, Naja naja kaouthia, Pseudechis colletti). Von 24 dieser 39 Giftschlangenbisse wurde der Verlauf bzw. der Schweregrad der Symptome mitgeteilt: Zwei Bisse verliefen ohne Symptome, 10 mit leichten, 6 mit mittelschweren und 6 mit schweren Symptomen. Todesfälle gab es keine.

Pilze: Es ereigneten sich keine tödlichen, wohl aber vier schwere Pilzvergiftungen. Bei allen vier ging es um Knollenblätterpilze (Amanita phalloides): Bei zwei Fällen wurde Amanitin analytisch nachgewiesen, in einem Fall konnte die Pilzidentifikation durch einen Mykologen gesichert werden. Im letzten Fall blieb es beim Verdacht auf Knollenblätterpilzvergiftung. Alle machten den typischen Verlauf mit schwerer Gastroenteritis durch, und die ersten drei Fälle zudem ein fulminantes Leberversagen. Alle vier Patienten erholten sich, eine Lebertransplantation wurde nicht nötig.

Andere Noxen: Ein schwerer Fall trat nach Einnahme von Ethanol in Kombination mit Butylscopolamin und Diazepam auf (Koma). Weitere Fälle betrafen eine Rauchgasvergiftung mit einem $\mathrm{COHb}$-Wert von $15 \%$ und schweren pulmonalen Reizsymptomen, einen Augenspritzer mit Methylenblau, der zu einer schweren Kornealäsion führte, und einen Tetanus durch eine Hautwunde. Die Patientin musste mehrere Tage intubiert und beatmet werden, und sie erhielt das Tetanus-Antitoxin. 


\section{Zunahme akzidenteller Vergiftungen}

Die Anzahl der Beratungen des Tox-Zentrums zu akzidentellen Giftexpositionen [1] hat zwischen 2002 und 2008 um $19 \%$ auf 21818 zugenommen (Abb. 1). Diese Zunahme ist überproportional, da die Gesamtanzahl der Beratungen im selben Zeitraum nur um 0,8\% stieg, und die Beratungen zu beabsichtigten Vergiftungen sogar um 6,6\% zurückgingen. Da die Chemiesicherheit in der Schweiz insgesamt auf einem vergleichsweise hohen Niveau ist, überrascht diese Feststellung. Daher soll dieser Anstieg hier etwas genauer analysiert werden.

Der Anstieg der Beratungen zu akzidentellen Expositionen geht fast vollständig auf Anrufe durch Privatpersonen zurück (+21,2\%), während die Anrufe durch Ärzte nur geringfügig zunahmen $(+4,6 \%)$. Bei den betroffenen Patienten handelte es sich gleichermassen um Erwachsene ( $>16$ Jahre) und um Kinder (16,1\% vs. $20,3 \%)$. Besonders markant war die $\mathrm{Zu}$ nahme mit 35,4\% bei den Kleinkindern (<6 Jahre). Dies steht im Einklang mit der Tatsache, dass vor allem Expositionen im häuslichen Milieu zunahmen $(24,7 \%)$.

Bei den Noxengruppen war vor allem bei den akzidentellen Expositionen mit Medikamenten (+29,3\%), Haushaltprodukten $(+23,2 \%)$, Körperpflege und Kos-

\section{Abbildung 1}

Entwicklung der jährlichen Anzahl von Beratungen des Tox-Zentrums zu akzidentellen Giftexpositionen (1999-2008).

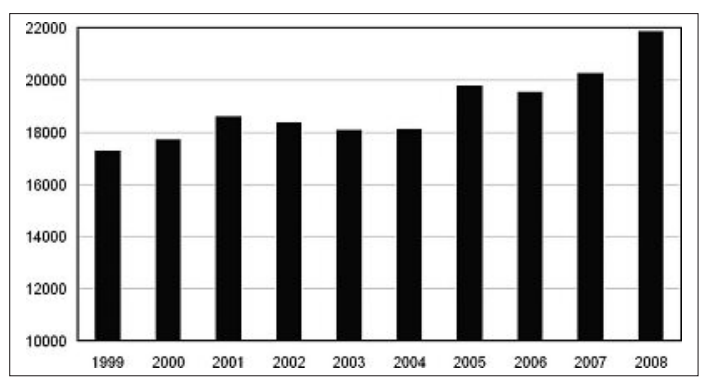

metika $(+36,2 \%)$ sowie mit Nahrungsmitteln $(+20,6 \%)$ ein Zuwachs zu verzeichnen, während es bei den Stoffen in Landwirtschaft und Gartenbau zu einem Rückgang von 5,2\% kam, und Pflanzenexpositionen sich gar nicht veränderten $(+0,6 \%)$. Diese Verteilung auf die Noxengruppen trifft sowohl für Erwachsene als auch Kinder zu (Tab. 3).

Für die Analyse der am meisten betroffenen Noxen wurde die absolute Zahl sowie der absolute und prozentuale Zuwachs der Fälle im Vergleich mit 2002 berücksichtigt, jeweils für 2008 und im Durchschnitt der Jahre 2003 bis 2008. Bei den Medikamenten verzeichneten akzidentelle Expositionen mit Paracetamol den grössten absoluten Zuwachs (+269 Fälle im Jahr 2008, je hälftig bei Kindern und Erwachsenen [2]), gefolgt von Quetiapin (+236), Cholecalciferol (+134), Zolpidem (+67), Ibuprofen (+63), Methylphenidat $(+56)$ und Lorazepam $(+53)$. Der grösste prozentuale Zuwachs war bei Oxycodon, Kaliumjodid und Sildenafil festzustellen, obschon die absoluten Zahlen dort sehr klein sind (Zuwachs durchschnittlich $<10$ Fälle). Bei den Haushaltprodukten nahmen die Beratungen zu Expositionen mit Geschirrreinigungsmitteln absolut am stärksten zu (+306 Fälle im Jahr 2008, davon 268 bei Kindern), gefolgt von anderen Reinigungsmitteln und Entkalkern. Der grösste prozentuale Zuwachs war bei den flüssigen Brennstoffen (Lampenöle, Anzündflüssigkeiten) festzustellen (Zuwachs absolut <25 Fälle). Bei den Körperpflegemitteln und Kosmetika nahmen Beratungen zu Seifen und Badezusätzen absolut am meisten zu, während die Mundpflegemittel den grössten prozentualen Zuwachs verzeichneten.

Im Bezug auf den Schweregrad der Vergiftungen kann aufgrund der kleinen Zahlen kein eindeutiger Trend festgestellt werden; die Zahlen in den Jahren 2002 bis 2008 liegen in der Schwankungsbreite. Diese Zahlen erlauben keine eindeutige Erklärung für die Zunahme der Beratungen zu akzidentellen Giftexpositionen. Neben einer tatsächlichen Zunahme der Expositionen selbst besteht die Möglichkeit, dass die Beratungen des Tox-Zentrums zunehmend in Fällen beansprucht werden, in denen früher keine Beratung gesucht wurde. Dies erachten wir gerade bei den akzidentellen Überdosierungen mit Paracetamol aber als eher unwahrscheinlich. Auch würde man dann erwarten, dass bei Vergiftungen mit allen Noxen gleichermassen häufiger angerufen wird. Dass die Häufung eine Folge der neuen Produktekennzeichnung bei Chemikalien ist, scheint eher unwahrscheinlich, weil nicht nur Haushaltprodukte, sondern auch Medikamente von der Zunahme betroffen sind. Da das Risiko eines Vergiftungsunfalles vor allem vom Zugang zu den Giften bzw. von deren Verfügbarkeit abhängt, und da fast ausschliesslich Anfragen zu Vergiftungsunfällen im häuslichen Milieu zunehmen, scheint es, dass mit Giften im Haushalt sorgloser umgegangen wird. Dies wäre ein Grund, die Vergiftungsprävention zu verstärken. 